\title{
Etude de la néologie terminologique en Bantu: cas de la cilubalisation des termes français des équipements et matériels de bureau'
}

\author{
Gilbert Kadima Batumona Adi
}

Département des Langues et Cultures, Université de Gand, Belgique

\begin{abstract}
"L'étude de la néologie terminologique en bantu ", à laquelle nous avons donc consacré notre thèse de doctorat en langues et cultures africaines, a eu pour point de départ des observations passives mais soutenues que nous avons faites sur de nombreux cas en rapport avec le recours aux technologies occidentales à des fins de modernisation d'un secteur de la vie socioculturelle au Congo-Kinshasa : l'administration publique. Ces technologies occidentales non localisées et fonctionnant dans leurs langues d'origine, connaissent à leur arrivée en République démocratique du Congo une situation de sous-utilisation essentiellement due à une carence terminologique dont font montre les langues de ce pays d'accueil, lesquelles (langues) ne sont pas suffisamment aptes à prendre les (technologies) en charge. Nous fondant sur de riches enseignements issus de l'analyse de cette situation atypique, dans la problématique de notre thèse, nous avons fait un constat que cette modernisation de l'administration assistée avec le recours aux technologies d'importation pose, en République démocratique du Congo, un réel problème de portée organisationnelle, économique et linguistique à la fois. Les raisons qui motivent ces conclusions sont fondées sur le fait que parmi de nombreuses difficultés qu'éprouvent les utilisateurs congolais de ces technologies, figurent non seulement des difficultés de savoir en faire usage, mais également celles de trouver des termes appropriés, susceptibles de les nommer dans leurs propres langues. C'est dans ce contexte que nous avons relevé le lien entre l'économie et la linguistique et partant de cette dernière discipline, nous avons également découvert la nécessité d'une recherche néonymique devant aboutir à l'élaboration d'un outil lexicographique ou terminologique multilingue, susceptible de soutenir les utilisateurs ciblés quant aux efforts d'appropriation de ces technologies dans leurs propres langues.
\end{abstract}

Mots Clés : Néologie, terminologie, traduction, aménagement linguistique, langues africaines bantu, ciluba, développement de langues peu dotées, équipements et matériels de bureau, linguistique appliquée 


\section{A study of neologisms in Bantu terminology: the case of Cilubalisation of French terms of office equipment and materials}

The Democratic Republic of Congo, like many African countries, has an important public and private administration, but one whose performance and productivity are generally considered to be low. Numerous efforts to modernize and improve the productivity of this administration have been made, notably by recourse to modern office equipment and supplies. In this doctoral study we label the equipment and supplies under the denomination of western technologies which signals their provenance. A close examination of the documents which catalogue and describe the functioning of these technologies, reveals that the overwhelming majority of these documents are written in either French or in English. In other words, the western technologies which are used in the Congolese public administration are denominated in the idioms of theirs designers and their operations are described in the same idioms. These technologies which range from simple to sophisticated tools (equipment for word processing or for electronic transmission of data) can be considered as the symbols of modern culture which generally is dominated by the western countries and some industrialized nations of Asia (Korea, Japan, Taiwan). The technologies are objects that are foreign to the cultures of Africa and the Congo. With regard to this situation, we argue that these imported western technologies must be localized when they arrive in African countries. To be localized means that the relevant documents be translated into the languages of the host countries in order to improve the efficiency with which the users are able to use the technologies. Our research demonstrates that when these imported technologies arrive in the Democratic Republic of Congo they are frequently under-utilized. This is because the occidental languages predominate in the operation documentation which are oriented for use within the social and legal contexts of western countries. Our analyses indicate that this under-utilization is essentially due to a terminological deficiency based on an assumption that the Congolese national languages are not sufficiently able to support the imported technologies. Our analyses also show that this assisted modernization of public administration in the Democratic Republic of Congo with the recourse to western technologies simultaneously poses organizational, economic and linguistic problems. Our conclusions not only point to operation difficulties but also to difficulties operators have finding the correct terms, which makes it more likely that items will be named in their own languages. It is in this context that we identify the link between economics and linguistics. From a linguistics position, the need of multilingual terminological research is clear, research that supports the users in the efforts to adapt their languages as languages of reception for these imported technologies. Eager to explore this field in connection with the Bantu languages and cultures we began our research for the thesis. Our thesis is articulated around four following activities: (i) development of the nomenclature having to be used as a sample of the study; (ii) the globalization or the methodological route of the terminological appropriation of western technologies which are addressed by our research; (iii) the study of the denominating terms of the aforementioned technologies; (iv) the study of the mechanism of an apparatus with regard to technical terms in Ciluba. The four activities outlined above will be developed over a planned eight chapters which constitute our thesis. This approach provides the focus for our research objectives, all of which are informed by one overriding need: providing the ciluba with one specialized glossary of office equipment and supplies.

Key words: Neology, terminology, translation, language planning, Bantu African languages, Ciluba, development of resourced languages, equipment and office supplies, applied linguistics 
"Les sciences et les techniques connaissent de nos jours une croissance exponentielle dans tous les domaines, si bien qu'il est devenu nécessaire de développer méthodiquement les vocabulaires qui s'y rapportent. Cela se fait maintenant couramment dans toutes les langues des pays développés, dans le cadre d'une nouvelle discipline académique, la terminologie ".

Diki-Kidiri (2008. postface)

\section{Objet de la recherche}

Il existe plusieurs définitions qui se rapportent au mot " néologie ». Celle qui suit et qu'en donne Le Petit Larousse illustré (2009) nous a semblé être un compromis, car elle se recoupe et se rapproche de beaucoup d'autres définitions qu'en donnent les spécialistes : " ensemble des processus de formation des néologismes (dérivation, composition, siglaison, emprunt, etc.)".

Dans notre thèse de doctorat, il est particulièrement question de la néologie terminologique, également appelée néologie dénominative, néologie traductive ou tout simplement néonymie selon sa désignation économique (que lui consacre Rondeau : I984). Quel que soit le vocable employé pour la désigner, ce qui importe de garder à l'esprit est sa particularité qui la singularise ainsi des autres néologies. En effet, selon ce qu'en note Guilbert (1975: 40), cette singularité réside non pas dans la volonté d'innover sur le plan de la langue, mais dans la nécessité de donner des noms à des objets afin de communiquer de nouvelles expériences.

Ainsi donc, démontrer comment devant cette nécessité de communiquer de nouvelles expériences et de dénommer entre autres les technologies occidentales, les communautés africaines les plus reculées peuvent se forger leurs propres désignations, est au centre ce que nous avons convenu d'appeler la cilubalisation. Ce mot (cilubalisation) est un néologisme que nous nous sommes forgés aux fins de donner une coloration africaine à cette démarche empreinte de modernité occidentale. En tant que démarche, la cilubalisation dans notre thèse de doctorat a consisté en la réflexion sur les termes dénommant les équipements et les matériels de bureau (EMB) et en un ensemble de méthodes aménagées aux fins de produire des terminologies appropriées en rapport avec ces technologies occidentales, exogènes à nos cultures luba-congolaises.

\section{Objectifs visés}

a) Démontrer que la connaissance ainsi que la maîtrise des procédés de création lexicale constituent les clés de succès dans toute entreprise d'enrichissement terminologique de nos langues nationales congolaises véhiculaires comme bien d'autres langues bantu en développement d'autant plus que c'est par le truchement de cet enrichissement que se réalise tout processus d'appropriation de la nouveauté et ce, quelle que soit son origine;

b) Étudier les matrices terminogéniques des termes " cilubalisants " du lexique des EMB c'est-à-dire les procédés à la base de leur fabrication et ce, en vue d'en dégager les spécificités ;

c) Contribuer aux recherches théoriques tant en aménagement terminologique, en terminologie descriptive qu'en néologie des langues bantu. 


\section{Structure}

Soucieux d'explorer ce nouveau domaine de recherche impliquant les langues bantu, nous avons, à cette fin, articulé notre dissertation doctorale autour de quatre activités ci-après : de prime abord, l'élaboration de la nomenclature devant servir d'échantillon de l'étude ; ensuite la " cilubalisation " ou l'itinéraire méthodologique de l'appropriation terminologique des technologies en présence ; puis l'étude des traits linguistiques des termes dénommant lesdites technologies et enfin l'étude des mécanismes de la création des termes en cilubà.

La mise en application de ces quatre activités a fonctionné comme dispositif de clarification de huit chapitres ayant constitué l'ossature de la dissertation doctorale et cette démarche a eu l'avantage d'être une balise qui a conduit vers des objectifs visés par notre recherche. Ces objectifs se résument en un " besoin ", celui d'outiller le cilubà d'un lexique spécialisé des termes dénommant les EMB et ce, en vue de son adaptation à l'expression de la modernité.

Les " données générales " constituent le premier chapitre et en tant que chapitre introductif ou l'entrée dans la matière, nous y avons présenté successivement la problématique de la recherche, l'objet d'étude de la thèse, l'hypothèse de la recherche, l'intérêt du sujet de la thèse, les objectifs visés par la thèse, l'orientation théorique de la thèse ainsi que son articulation.

Nous avons jugé utile de consacrer quelques lignes aux faits socio-historiques en rapport aussi bien avec le ciluba qu'avec la cilubalisation dans le deuxième chapitre auquel nous avons donné le titre de repères historiques et sociolinguistiques. Dans ces repères, tout en revenant sur les origines du peuple luba et de sa langue, nous y avons également montré que les efforts de modernisation ou d'enrichissement du ciluba ne sont pas des faits nouveaux, étant donné qu'ils remontent aux premiers contacts que ses locuteurs ont eu avec d'autres peuples et cultures.

"Tout linguiste qui veut décrire le langage et les langues se doit de commencer toujours par quelques données sur les langues ", rappelle Cabré (2000 : I4). Ces données sur les langues constituent, dans le jargon du linguiste, ce qu'on a pris l'habitude d'appeler le corpus. Celui-ci consiste en une documentation disponible sur le domaine de recherche ou en un ensemble de données sur la langue à l'étude. C'est par le truchement de cette documentation ou de ces données que le linguiste peut parvenir aux objectifs visés par son analyse et c'est en vue de nous conformer aussi à cette exigence méthodologique que nous avons consacré le troisième chapitre de notre étude au corpus ainsi qu'aux outils méthodologiques.

Le quatrième chapitre décrit les fondements théoriques de la néonymie ou de la néologie (en contexte) teminologique. Pour y parvenir, les principes et les notions de base des disciplines à l'origine de la néologie terminologique sont de prime abord passés en revue afin de mieux replacer ce type de néologie dans son contexte d'apparition et ce, avant de les appliquer à la localisation en ciluba des EMB. Aux fins de mener à bien notre entreprise, expliciter les notions de base et les questions de méthode, nous nous sommes référé aux écrits et ouvrages de base en la matière, notamment ceux de Diki-Kidiri (2008), Rey (1979), Cabré (2007), 
Dubuc (1978,1980), Boutin-Quesnel (1990), Kreckova (1997), Gouadec (1990), Bouveret (1996), L'Homme (2004) ainsi que L'Homme et Vandaele (2007).

Le cinquième chapitre est un condensé d'éléments grammaticaux et lexicologiques du ciluba en rapport avec notre recherche doctorale. Son objectif est : (i) d'aider les lecteurs non luba et non linguistes à comprendre les règles grammaticales qui président à la formation des mots en ciluba, et (ii) de se familiariser surtout avec la terminologie grammaticale que nous utilisons dans le sixième chapitre, consacré à l'analyse morphosémantique des termes cilubalisant/dénommant les EMB.

La problématique envisagée dans le sixième chapitre a porté sur la création des termes techniques du ciluba en rapport avec les lois morphologiques, structures syntaxiques (matrices lexicogéniques) ou mécanismes sémantiques auxquels ces termes obéissent. Nous sommes parti du morphème comme unité d'analyse et notre tâche a consisté à démontrer la manière dont cette unité est impliquée aussi bien par sa forme que par son sens dans la fabrication des termes en bantu.

Dans le septième chapitre, (i) nous nous sommes intéressé aux mécanismes et procédés de création néologique. Ici, notre tâche de linguiste bantuiste à consisté à scruter toutes les méthodes et tous les processus que nous avons mis en œuvre lors de la fabrication des termes ; (ii) nous avons outre inventorié les matrices terminologiques propres au domaine à l'étude et (iii) nous avons terminé (le chapitre) par des questions relatives à la dynamique et au poids des termes dénommant les EMB.

Le mot " terme ", rappelons-le en passant, est la dénomination d'un concept en une langue spécialisée. Les expressions "langue spécialisée ", "langue de spécialité " ou « langue en spécialité " ne désignent rien d'autre que la langue naturelle en situation d'emploi professionnel, c'est-à-dire une langue naturelle utilisée pour techniquement rendre compte de connaissances spécialisées (Lerat, 1995 : 21). Dans le cadre de notre recherche doctorale, les termes réunis pour rendre techniquement compte des connaissances spécialisées propres aux EMB sont tous des appellations d'emploi.

Le huitième et dernier chapitre de notre recherche est consacré à la conclusion générale. Celle-ci précède la bibliographie, les parties annexes et surtout les index qui, comme d'ordinaire, viennent en fin des travaux de recherche à caractère terminologique. Comme dans tout travail de ce genre, notre conclusion générale a eu, entre autres pour tâches de récapituler les points saillants de nos analyses, de montrer en quoi les objectifs visés par notre recherche ont été soigneusement atteints et en quoi la recherche a constitué une contribution aux études portant sur la néonymie et enfin, d'identifier les perspectives à venir que notre recherche pourrait ouvrir.

\section{Orientation théorique}

Étant donné que la terminologie peut être envisagée selon quatre aspects : la politique linguistique, la normalisation, l'aménagement terminologique et la théorie terminologique, dans le cadre de notre recherche doctorale, nous avons décidé de l'étudier sous son aspect d'aménagement et d'équipement terminologique. Afin de mener à bien cette entreprise, nous avons décidé de baser notre recherche sur l'approche systématique de la terminologie. 
Cette approche procède d'ordinaire par des ensembles de termes qui appartiennent à un domaine de spécialité et qui sont obtenus grâce à un dépouillement de textes représentatifs du domaine à l'étude et s'effectue par la comparaison entre deux ou plusieurs langues (Corbeil, s.d.). Nous avons complété cette approche avec une autre dénommée " approche culturelle de la terminologie ". La particularité de cette dernière tient au fait qu'

" elle met l'héritage culturel de ceux qui s'approprient les nouveautés au centre de tous les modes linguistiques de désignation de nouveaux termes au lieu que ces modes soient calqués sur les modèles linguistiques des cultures d'origine des nouveautés. Cet héritage culturel qui se nourrit de toute l'expérience humaine en termes de productions, de savoirs et savoirs-faire perçoit et re-conceptualise chaque nouveauté en y intégrant la culture et devient une sorte d'archétype ou de grille d'interprétation pour la compréhension et l'appropriation de nouvelles réalités " (Diki-Kidiri, $2000: 6)$.

Une telle démarche préserve non seulement le respect des identités des communautés les moins outillées désireuses de s'approprier de nouvelles technologies, mais elle conditionne et préserve surtout le succès de leur intégration à l'héritage culturel.

\section{Démarche}

De plus en plus, on conçoit la terminologie comme un domaine de connaissance nécessairement interdisciplinaire qui intègre aussi bien les aspects cognitifs, linguistiques, sémiotiques que communicatifs des unités terminologiques (Cabré, 2000 : 13). Étant donné que nous ne pouvions pas entreprendre une recherche traitant de toutes ces dimensions, au cours de nos recherches, nous nous sommes senti obligé de préciser les aspects que nous devrions traiter et nous avons opté pour les aspects linguistiques, étant donné que comme Cabré (2007: 85), nous soutenons que les termes comme ceux dénommant les EMB (à l'étude) sont des signes linguistiques.

C'est dans ce contexte que dans le quatrième chapitre de notre dissertation, après avoir mentionné que la terminologie était la discipline scientifique au sein de laquelle nous inscrivions notre recherche, nous nous étions également senti obligé de préciser que c'était la porte linguistique qui devait nous y conduire.

Pour y parvenir, la démarche que nous avons suivie a consisté en la clarification de quatre activités assignées à notre recherche, à savoir : de prime abord, l'élaboration du corpus ; ensuite la localisation ou mieux la cilubalisation c'est-à-dire la traduction des termes français dénommant les EMB ou les technologies en présence ; puis l'étude des traits linguistiques des termes dénommant lesdites technologies et enfin l'étude des mécanismes de la création des termes en cilubà.

\subsection{L'élaboration du corpus}

Il n'est pas d'étude linguistique ou littéraire qui puisse se faire sans corpus et il a été dit que " tout linguiste qui veut décrire le langage et les langues commence toujours avec quelques données sur les langues " (cf. Cabré, 2000 :I4)! Pour sa part, Dubois (1969: 
II7) voit le " corpus " comme l'ensemble déterminé des textes auxquels on applique(ra) des méthodes définies. Ne pouvant pas déroger à cette règle, nous nous étions également constitué un corpus aux fins de faire avancer notre problématique.

En effet, dans notre recheche doctorale, le corpus auquel nous avons eu à appliquer nos méthodes est basé sur notre publication intitulée " Lexiques ciluba-français-cilubà des équipements et matériels de bureau " dont les détails bibliographiques sont mentionnés dans la bibliographie (Kadima, 2007). Cette dernière publication est une mise à jour de la version multilingue élargie aux lingala et kikongo, publiée sous le label du CELTA en 2002.

Lélaboration ainsi que la mise à jour a été rendue possible grâce au dépouillement de plusieurs documents à caractères terminographiques dont les plus importants sont ceux de ces auteurs :

a) Corbeil, Jean-Claude et Anane Archambault, (1992), Le Visuel : dictionnaire thématique français-anglais, zè édition, Montréal, Editions Québec/Amérique, 896 pages.

b) Bureau des traductions. 1985. Fournitures de bureau. Lexique français - anglais - français, publié par la Direction de la terminologie, Bureau des traductions, Secrétariat d'État, Ottawa (Ontario), 23 pages.

c) Ginguay Michel. 1990. Dictionnaire d'informatique, bureautique, télématique, anglais - français, Paris Masson, Ioè édition, 26o pages.

d) Gulloton Noëlle et Hélène Cajolet-Laganière. 1996. Le français au bureau, Les Publications du Québec, 400 pages (Guides de l'Office de la langue française).

\subsection{La cilubalisation}

Nous avons utilisé le néologisme cilubalisation dans notre recherche doctorale pour désigner trois importantes choses : l'étape centrale dans la constitution de la nomenclature en langue-cible (ciluba) ; l'activité de création en ciluba des nouvelles unités lexicales; une grille de procédés néologiques. Les deux premières ont été abordées dans ce troisième chapitre (corpus et outils méthodologiques) tandis que la dernière l'est au septième chapitre (néologie et mécanismes de création des termes des EMB).

En tant qu'activité principale assignée à notre recherche, la cilubalisation a consisté essentiellement dans la traduction ou en l'adoption en ciluba des réalités venant d'autres langues ou cultures aux fins d'obternir des termes qui respectent les traditions et les régles d'ordre phonétique ou grammatical du ciluba.

Nous avons mené cette laborieuse tâche de traduction dans le strict respect des principes de traduction que rappellent Hermans et Vansteelandt (1999: 37-43) dans leur brillante étude. Ces trois principes sont : l'équivalence du message, le respect des traditions terminologiques de la spécialité et le respect du génie de la langue cible. Ci-dessous, nous illustrons ces trois principes par des exemples. 
- L'équivalence du message

La traduction étant fondée sur le principe de l'équivalence du message entre la langue de départ et la langue d'arrivée, nous pouvons, en partant de ce principe, dire que la nomenclature en langue d'arrivée, résultante de la traduction, a la même fonction que la nomenclature en langue de départ, car son objectif est de transmettre les mêmes informations, disons mieux le même message. Ce message, comme celui de la langue de départ, est destiné à un même utilisateur et se caractérise par sa nécessaire immédiatété avec la réalité.

\begin{tabular}{l|l} 
Langue source/français & Langue cible/cilubà L/3ra \\
\hline les instruments pour écrire & bifûndidi \\
\hline les instruments pour couper & bikòsedi \\
\hline les instruments pour coller & bilamikijiji \\
\hline les instruments pour classer & bilongamijiji
\end{tabular}

- Le respect des traditions terminologiques de la spécialité

Ce principe voudrait que le traducteur ou mieux la traduction rendue, en sus de la correspondance du message, respecte également les traditions qui président à la création des termes dans la langue cible. Les exemples que contient le tableau suivant montrent nos méthodes de traduction qui sont respectueuses de ce principe.

\begin{tabular}{l|l|l} 
Ciluba L3ra & Français & Modèle et traduction \\
\hline \multirow{2}{*}{ lùshoolà } & ciseaux, cisailles & cyamù = appareil, instrument, engin \\
\cline { 3 - 3 } & & màshinyì = engin avec moteur, machine \\
\hline $\begin{array}{l}\text { lushoolà lwà (dikòsa } \\
\text { naalù) mabèji }\end{array}$ & ciseaux à papier & cyamù cyà (dikomena) bilàmbà \\
\cline { 3 - 3 } $\begin{array}{l}\text { lushoolà lwà (dikòsa } \\
\text { naalù bintu mu) bì̀lo }\end{array}$ & ciseaux de bureau & fer à repasser \\
\cline { 3 - 3 } & & cyamù cyà makàla \\
\cline { 3 - 3 } & & fer à repasser à braise
\end{tabular}

- Le respect du génie de la langue cible

L'observation de ce principe veut que le traducteur demeure conservateur et suive, dans la création de ses termes, les voies tracées par la langue cible dont il doit respecter le génie. Les termes auxquels il donne vie se doivent d'être motivés.

Notre conservatisme quant au respect du génie de la langue ciblée est également manifeste à travers nos exemples qui, comme nous les voyons, sont presque tous motivés. En effet, les classes nominales ci-/bi sont connues en ciluba comme celles qui englobent les lieux et surtout les instruments. A cette marque descriptive, distinctive ou sémantique liée aux classes $5 / 6$ du ciluba, aux fins de renforcer l'idée ou la notion d'outil, nécessité ou instrument, vient s‘ajouter le suffixe applicatif (-il-, -el-, -in-, -en-) qui tra- 
duit l'instrument qui sert aux diverses actions (couper, attacher, coller, corriger, classer, attacher, etc.).

\begin{tabular}{l|l|l} 
Français & Message & Ciluba $\mathrm{L}_{3} \mathrm{I}_{\mathrm{a}}$ \\
\hline fourniture & outil, instrument, nécessité & lu/nkèngelu \\
\hline $\begin{array}{l}\text { fournitures de } \\
\text { bureau }\end{array}$ & nécessité(s) au travail de bureau & nkèngelu ya midimu yà bìlò \\
\hline $\begin{array}{l}\text { instruments pour } \\
\text { attacher }\end{array}$ & nécessité(s) pour attacher & byashi \\
\hline $\begin{array}{l}\text { instruments pour } \\
\text { classer }\end{array}$ & nécessité(s) pour classer & bilongamijiji \\
\hline $\begin{array}{l}\text { instruments pour } \\
\text { coller }\end{array}$ & nécessité(s) pour coller & bilamikijiji \\
\hline $\begin{array}{l}\text { instruments pour } \\
\text { corriger }\end{array}$ & nécessité(s) pour corriger & byakajiji \\
\hline $\begin{array}{l}\text { instruments pour } \\
\text { couper }\end{array}$ & nécessité(s) pour couper & bikòsedi
\end{tabular}

\subsection{L'étude des traits linguistiques}

Cette étude s'est déroulée au sixième chapitre (analyse morphosémantique des termes cilubalisant les EMB) de notre recherche et porte systématiquement sur toutes les $66_{3}$ UT. Les différentes étapes de la méthode suivie dans ce chapitre auquel nous avions assigné comme objectif principal de justifier par l'analyse morphosémantique, l'attribution des termes cilubalisant ou dénommant les EMB sont :

- la présentation de la vedette c'est-à-dire du terme " cilubalisant " et de sa correspondante en langue source ;

- la décomposition (si possible) de la vedette en morphèmes, et avec précision de toutes les modifications morphologiques ;

- la déclinaison pour chacun des morphèmes du " cilubalisant " de sa nature grammaticale et le cas échéant d'une possible signification ;

- l'indication pour le classificateur ou préfixe de sa classe nominale et précision pour la base c'est-à-dire le radical de son origine ou de son infinitif s'il s'agit d'un verbe ;

- l'indication pour chaque morphème dérivatif (suffixe) de son apport sémantique ;

- la traduction française du terme " cilubalisant " et ce, après sa déclinaison, et tout en nous appuyant sur son classificateur, son radical ainsi que sur ses autres morphèmes dérivatifs (suffixes).

Quelques exemples des cilubalisants analysés sur le modèle de ceux que nous avons mentionnés précédemment en parlant du respect du génie de la langue cible ainsi que celui des traditions terminologiques de la spécialité peuvent aider nos lecteurs à comprendre comment cette analyse s'est déroulée. 
Exemples :

nkèngelu yà midimu yà bì̀lò : fournitures de bureau

n-kèng-el-u i-à mi-dimu i-à $\emptyset$-bì̀lò

$\mathrm{n}$ - : préfixe nominal de classe 9

-kèng- : théme nominal déverbatif de kukènga = éprouver une nécessité

-el- : suffixe dérivationnel applicatif

$-\mathrm{u}$ : suffixe dérivational final

i- : préfixe d'accord du déterminatif de destination

-à : suffixe dérivationnel final

mi- : préfixe nominal de classe 4

-dimu : thème nominal $=$ travail

i- : préfixe d'accord du déterminatif de qualification

-à : suffixe dérivationnel final

$\emptyset$ - : préfixe nominal de classe zéro

-bìlò : théme nominal d'emprunt au français = bureau

Traduction française : les nécessités qui sont/qui servent pour le travail de bureau

byashidi : instruments pour attacher

bi-as-il-i

bi- : préfixe nominal de classe 8

-as- : thème nominal déverbatif de kwasa = bâtir, attacher

-il- : suffixe dérivationnel applicatif

-i : suffixe dérivationnel final

Traduction française : les instruments qui sont/qui servent à attacher

bilamikijiji : instruments pour coller

bi-lam-ik-ij-ij-i

bi- : préfixe nominal de classe 8

-lam- : thème nominal déverbatif de kulama = garder

-ik- : suffixe dérivationnel passif

-ij- : suffixe dérivationnel applicatif

-ij- : suffixe dérivationnel applicatif

-i: suffixe dérivationnel final

Traduction française: les instruments qui sont/qui servent à coller 
byakajiji : instruments pour corriger

bi-akaj-ij-i

bi- : préfixe nominal de classe 8

-akaj- : thème nominal déverbatif de kwakaja = corriger, arranger

-ij-: suffixe dérivationnel causatif

-i : suffixe dérivationnel final

Traduction française: les instruments qui sont/qui servent à corriger

bikòsedi : instruments pour couper

bi-kòs-el-i

bi- : préfixe nominal de classe 8

-kòs- : théme nominal déverabtif de kukòsa = couper

-el- : suffixe dérivationnel applicatif

$-i$ : suffixe dérivationnel final

Traduction française : les instruments qui sont pour couper/qui servent à couper

bilongamijiji : instruments pour classer

bi-long-am-ij-ij-i

bi- : préfixe nominal de classe 8

-long- : thème nominal déverbatif de kulonga = classer, disposer, ranger

-am- : suffixe dérivationnel statif

-ij- : suffixe dérivationnel applicatif

-ij- : suffixe dérivationnel applicatif

$-i$ : suffixe dérivationnel final

Traduction française : les instruments qui sont pour ranger, classer ou disposer

Toutes nos 663 UT aussi bien simples que complexes ont subi un traitement semblable, celui d'avoir été découpées et analysées morphologiquement. L'objectif poursuivi par cette analyse et cette découpe était de rechercher toutes les unités de forme pourvues d'un sens, c'est-à-dire les " morphèmes ". Cette analyse, quoique laborieuse, a eu le mérite de mettre à découvert les capacités des morphèmes qui, structurent notre manière d'exprimer la réalité et participent en même temps à la construction du sens ainsi qu'à la fabrication du terme à travers divers procédés néologiques.

5.4. L'étude des mécanismes de la création des termes en cilubà

Cette étude s'est déroulée dans le développement du septième chapitre (néologie et mécanismes de création des termes des EMB). Par le truchement de ce chapitre, nous visions à repertorier tous les moyens linguistiques et néologiques qui nous ont servi à titre d'outils pour l'élaboration ou la création de nos $66_{3}$ UT. 
Tous les langagiers savent que la formation des nouvelles unités terminologiques (UT) est réalisée par le recours aux mêmes procédés de formation des mots de la langue 'générale et que chaque langue a ses propres moyens linguistiques dont elle se sert pour créer des dénominations (Kreckova, I997 : 68). En ce qui nous concerne, les moyens linguistiques que nous avons mis en œuvre lors de la création de nos UT sont synthétisés dans le tableau qui va suivre qui fait état de la grille néologique que nous avons mise au point aux fins de servir d'outil de description de langues spécialisées du domaine bantu.

\begin{tabular}{|c|c|c|c|c|c|}
\hline \multirow{10}{*}{ matrice interne } & \multirow{7}{*}{$\begin{array}{l}\text { néologie } \\
\text { de forme }\end{array}$} & \multirow{6}{*}{ construction } & \multirow{3}{*}{ affixation } & \multirow{6}{*}{$\begin{array}{c}\mathrm{m} \\
\mathrm{i} \\
\mathrm{x} \\
\mathrm{a} \\
\mathrm{t}\end{array}$} & préfixation \\
\hline & & & & & suffixation \\
\hline & & & & & $\begin{array}{l}\text { formation } \\
\text { parasynthétique }\end{array}$ \\
\hline & & & \multirow{3}{*}{ composition } & & juxtaposition \\
\hline & & & & & synapsie \\
\hline & & & & & $\begin{array}{l}\text { formation } \\
\text { syntagmatique }\end{array}$ \\
\hline & & \multicolumn{2}{|c|}{ imitation et déformation } & $\mathrm{i}$ & onomatopée \\
\hline & \multirow{3}{*}{$\begin{array}{c}\text { néologie } \\
\text { de sens }\end{array}$} & \multirow{3}{*}{\multicolumn{2}{|c|}{ changement de sens }} & 0 & métaphore \\
\hline & & & & $\mathrm{n}$ & métonymie \\
\hline & & & & & $\begin{array}{l}\text { extension, restriction } \\
\text { de sens }\end{array}$ \\
\hline matrice externe & \multicolumn{3}{|c|}{ néologie par emprunt } & & emprunt \\
\hline
\end{tabular}

La mise de cette grille a été entre autres motivée par le fait que nous avons remarqué que la typologie des procédés néologiques en usage sur les langues congolaises semblait dépassée et qu'elle ne tient pas compte des progrès accomplis en linguistique et plus particulièrement en matière d'élaboration des vocabulaires techniques. La science étant donc faite de continuelles remises en question et soucieux d'obtenir des nouvelles unités terminologiques qui respectent les traditions ainsi que les règles d'ordre phonétique ou grammatical du ciluba et qui tiennent compte de ces progrès, nous avons senti la nécessité de disposer de notre propre typologie des procédés néologiques.

Affixation

L'affixation ou la dérivation est donc un procédé de création lexicale qui consiste à fabriquer de nouvelles lexies en usant d'éléments lexicaux comprenant essentiellement des radicaux ainsi que des affixes. C'est un procédé très productif dans les langues de spécialité (Hermans, I995: I2). 
En cilubà, comme dans beaucoup de langues du domaine bantu, par l'adjonction d'un préfixe ou d'un suffixe à une base, nous obtenons un mot dérivé. Dans cette langue à l'étude, le ciluba, l'affixation répond à la structure suivante : préfixe + base + suffixe $\rightarrow$ lexie dérivée.

Préfixation

Il y a donc préfixation lorsque l'affixation s'opère par l'adjonction des préfixes.

Exemples :

\begin{tabular}{l|l|l|l} 
ku-bànd-ish-a & faire monter & -bànd- & ka-bànd-? \\
\hline ku-tàp-a & blesser & -tàp & n-tàp-? \\
\hline ku-bwikil-ij-a & couvrir & -bwikil- & ci-bwikil-? \\
\hline ku-fund-a & écrire & -fùnd & mu-fùnd-
\end{tabular}

Les ? indiquent les places réservées aux suffixes.

Suffixation

On parle de la suffixation lorsque l'affixation s'opère par l'adjonction des suffixes.

Exemples :

\begin{tabular}{l|l|l|l|l} 
ku-bànd-ish-a & faire monter & -bànd- & kabànd-ish-i & butée \\
\hline ku-tàp-a & blesser & -tàp & ntàp-a & tranchant \\
\hline ku-bwikil-ij-a & couvrir & -bwikil- & cibwikil-ij-i & couvercle \\
\hline ku-fund-a & écrire & -fünd & mufünd-u & texte
\end{tabular}

Formation parasynthétique

Il y a formation parasynthétique lorsqu'un substantif dérivé rassemble en lui les deux modes d'affixation dont il venait d'être question ci-haut, c'est-à-dire la préfixation et la suffixation.

Exemples :

\begin{tabular}{l|l|l|l|l|l|l}
$\begin{array}{l}\text { Verbe du } \\
\text { départ }\end{array}$ & Traduction & Préfixe & Radical & Suffixe(s) & Terme dérivé & Traduction \\
\hline kubàndisha & $\begin{array}{l}\text { faire mon- } \\
\text { ter }\end{array}$ & ka- & -bànd- & -ish-i & kabándishi & butée \\
\hline kutàpa & blesser & n- & -táp- & -a & ntàpa & tranchant \\
\hline kubwikila & couvrir & ci- & -bwikil- & ij-i & cibwikidiji & couvercle \\
\hline kufùnda & écrire & mu- & -fund- & -u & mufùndu & texte
\end{tabular}


Partant de ce principe (réunion dans un dérivé de la dérivation et de la suffixation) nous pouvons dire qu'en ciluba beaucoup de substantifs sont formés par ce procédé, étant qu'il n'est pas dans cette langue un seul substantif qui soit dépourvu d'affixes (préfixe et suffixe).

\section{Composition}

En linguistique le terme composition est un générique qui englobe selon nos investigations trois procédés distincts de création lexicale, à savoir : la juxtaposition, la composition synaptique (la synapsie) ainsi que la formation ou la composition syntagmatique.

Composition par juxtaposition

C'est un mode de composition dans lequel les éléments lexicaux de forme pleine avec ou sans trait d'union sont réunis en mot composé (Boutin-Quesnel, I990:24).

\begin{tabular}{l|l}
\multicolumn{1}{c|}{$\begin{array}{l}\text { Exemples : } \\
\text { cipeta-ndambù }\end{array}$} & total-partiel \\
\hline òrdìnàteer-mukesè & micro-ordinateur \\
\hline nsonga-kèlèyo & taille-crayon \\
\hline mbùta-byâla & signataire \\
\hline kabala-matùku & calendrier
\end{tabular}

\section{Composition synaptique}

La synapsie est mode de composition par lequel le déterminé est relié au déterminant par le truchement d'un mot de liaison couramment appelé connectif, joncteur ou déterminatif du point de vue fonctionnel.

Exemples :

\begin{tabular}{l|l} 
àkràfeez wa nzembu & agraffeuse électrique \\
\hline àmvilopà wa dìdìdishì & envelope-fenêtre \\
\hline àtashì wa dìmpìnda & attaché croisée \\
\hline bakà wa mikàndà & bac courrier \\
\hline bùlòkùnotà bwà brùyo & bloc-note brouillon
\end{tabular}

Formation syntagmatique

Nous entendons par la formation syntagmatique un mode de composition qui conduit à la formation d'un syntagme terminologique, c'est-à-dire un terme constitué d'une suite terminologisée de mots liés syntaxiquement et désignant une notion unique (Boutin-quesnel, I990 : 24) dans un domaine déterminé du savoir (l'ajout vient de nous). 
Exemples :

àkràfeez wa tukànu twà mihimbu

dilongolola dyà mfündilu

cibweledi cyà mabèji àà difwatwila

cilaminyi cyà àtashì yà bikwàtàkwàtà

Mixation

C'est le procédé de création des termes qui consiste à associer au sein d'une même unité lexicale les deux procédés d'affixation et de composition (Halaoui, I993: 710).

Exemples :

- Affixation + composition synaptique cilaminyi cyà àtashì yà cikwàtàkwàtà

- composition synaptique + composition par juxtaposition + affixation dikàpàkapà dyà cyalula mashìka

- composition synaptique + composition par juxtaposition + affixation kashèètà kàà mikàndà mikulukulu ya bilengejilu

- composition synaptique + composition par juxtaposition + affixation màshinyì mapandi àà mikàndá mipeepèlùka

- emprunt + composition synaptique + composition juxtaposition + affixation mbòta wa cifila mpùngà mishìlàshìlàngàna

\section{Extension de sens}

C'est un mode de formation de termes par lequel un terme existant est utilisé pour désigner une notion nouvelle et ce, en vertu d'un élargissement de son sens originel. Dans le vocabulaire systématique de la terminologie, Boutin-Quesnel (r99o: 25) note que l'extension de sens et l'emprunt interne se confondent à la limite. La première est perçue du point de vue notionnel (modification de la notion désignée par un terme existant) alors que la seconde est perçue du point de vue formel (utilisation d'un terme existant).

Exemples :

\begin{tabular}{l|l|l} 
Terme originel & Sens général & Sens élargi dans les EMB \\
\hline mbòta & bouton & touche (informatique) \\
\hline lumwenu & miroir & vitrine, écran \\
\hline mâyi & eau & encre \\
\hline mpaya & poche & soufflet
\end{tabular}

La métaphore

C'est un procédé néologique qui consiste à employer une unité lexicale existante en lui conférant un sens qu'il n'avait pas auparavant. 
Exemples :

\begin{tabular}{l|l|l|l} 
Terme & Sens originel & Sens métaphorique & Traduction \\
\hline kapùku & souris (animal) & souris (informatique) & calendrier \\
\hline cimònu & hanche (anatomie) & joint (ciseaux) & photographier \\
\hline mutù & tête (anatomie) & tête (imprimante) & lampe de table \\
\hline dìdìdiishì & fenêtre & volet (informatique) & administration \\
\hline cikwàcilu & poignet & anneau (ciseau) & microphone
\end{tabular}

\section{Emprunt}

En tant que procédé de création lexicale, l'emprunt consiste à incorporer dans une langue donnée un signe linguistique provenant d'une autre langue.

Exemples :

\begin{tabular}{l|l|l} 
Langue d'arrivée & Emprunt & Langue source \\
\hline faks & fax & français \\
\hline fiishì & fiche & français \\
\hline làzeer & laser & français \\
\hline òrdìnàteer & ordinateur & français \\
\hline skaneer & scanneur & français
\end{tabular}

Calque

C'est un type d'emprunt lexical particulier qui consiste en la traduction littérale d'une langue à une autre de l'unité lexicale empruntée.

Exemples :

\begin{tabular}{l|l|l} 
Terme originel & Langue source & Calque en ciluba \\
\hline calendrier & français & kabala-matùku \\
\hline kufotola & kiswahili & kufwatula \\
\hline lampe de tableau & français & mwêndù wa pamêsà \\
\hline mbulamatari & kikongo & mbùlàmàtadì \\
\hline microphone & français & cikòlùkòlù
\end{tabular}

\section{Résultats}

Comme le rapporte Café Ligia (1999 : 248) et de la même manière que l'avons mentionné dans la note introductive à notre sixième chapitre, l'étude des termes peut se faire selon plusieurs points de vue dont les plus traditionnels et les plus populaires sont : le point de vue formel qui s'intéresse au terme en tant que dénomination, le point de vue sémantique qui approche le terme comme concept et le point de vue fonctionnel qui considère le terme comme un objet d'une catégorie. 
Ainsi que nous pouvons nous en rendre compte au terme de ce compte rendu, notre recherche a traité essentiellement les 663 termes du point de vue de leur forme et est arrivée à la conclusion que le "morphème " joue un rôle des plus incontournables dans la fabrication de ces signes linguistiques dans les langues du domaine bantu. Cet incontournable rôle tient surtout au fait qu'en tant que formant, le morphème structure la manière d'exprimer la réalité et participe en même temps à la construction du sens du terme. Le septième chapitre de notre étude est également revenu sur cet aspect de la chose avec de longs commentaires ainsi que des détails supplémentaires.

$\mathrm{Au}$ terme de toutes nos analyses et à la lumière de tous les aspects linguistiques examinés, nous sommes arrivé à la conclusion que l'étude de la néologie terminologique en bantu, à l'exemple de celle que nous venons d'effectuer est sans conteste une étude essentiellement linguistique.

\subsection{Réponse}

Notre réponse, ici, ne se situe pas par rapport à une quelconque question à laquelle notre recherche doctorale se devait de répondre. Elle se situe par rapport à un " besoin ", entendu au sens "d'écart mesurable " existant entre " ce qui est " et " ce qui devrait être " tel que nous l'avons, de prime abord, appris au quatrième chapitre de Loubier (1994: 49) et tel que nous l'avons, par la suite, emprunté de cette auteure à des fins de son application au contexte tout particulier de cette recherche.

La répétition étant la mère des sciences, ainsi que nous l'avons souvent entendu dire, nous estimons qu'il est important, avant de montrer la manière dont cette recherche prétend avoir répondu à ce besoin, d'en rappeler la situation particulière. En effet, scrutant l'état de nos productions terminologiques, nous relevions d'une part que non seulement ces productions étaient loin de satisfaire les besoins terminologiques du Congo-Kinshasa, mais qu'elles ne répondaient pas non plus aux exigences de nouvelles théories en néologie terminologique. D'autre part, nous relevions en même temps que les mêmes productions ne devaient pas demeurer cloisonnées dans la seule activité ou pratique consistant en l'établissement des nomenclatures bilingues. C'est ce volet de la situation qui, en aménagement linguistique, correspond à " ce qui est ".

Quant à " ce qui devrait être ", c'est-à-dire la situation souhaitée et qui constitue le volet auquel cette recherche se trouve être une réponse est l'ouverture si pas l'amorce des études néologiques et terminologiques portant aussi bien sur les traits linguistiques des termes que sur leurs mécanismes de création, autrement-dit la terminologie descriptive qui marque une rupture avec nos traditions en matière de recherche terminologique.

En dehors de ce " besoin " sous son aspect qui vient d'être commenté, notre réponse se situe également par rapport aux trois objectifs principaux visés par notre thèse et qui ne sont pas à confondre avec des objectifs particuliers assignés à chacun de ses différents chapitres. En rapport avec ces derniers objectifs et vu sous l'angle de "ce qui devrait être ", en guise de réponse, nous aimerions ajouter que par ce travail, nous croyons avoir inauguré une nouvelle ère des recherches en néonymie appliquée aux langues nationales congolaises bantu. 
Particulièrement sur ce dernier point, nous disons que si ce travail peut être d'une quelconque utilité ou servir de modèle à tous ceux qui font des recherches terminologiques théoriques sur les vocabulaires techniques en bantu, c'est que ces recherches (jusqu'ici cloisonnées dans les élaborations des nomenclatures bilingues) auront pris certainement une autre dimension et franchi irréversiblement un nouveau pas. C'est ce qui constituait le troisième des objectifs généraux que visait la présente recherche.

Le moment est également venu d'aborder la question relative à la spécificité des termes dénommant les $\mathrm{EMB}$ en ciluba étant donné que toutes leurs matrices terminogéniques ont été soigneusement passées au crible. Mais avant d'y parvenir, il convient de rappeler ce que l'on entend par matrice terminogénique. En effet, une matrice terminogénique est un processus fondamental de création d'une nouvelle unité terminologique (UT). En termes plus clairs, une matrice terminogénique veut dire tout simplement un moyen néologique auquel le terminologue a recours en vue de la création d'un terme. En rapport avec notre recherche doctorale, les moyens néologiques auxquels nous avons eu recours aux fins d'élaborer les termes destinés à dénommer les EMB en ciluba sont : la mixation, la préfixation, la suffixation, la juxtaposition, la composition synaptique, la formation syntagmatique, la mixation, la métaphore, l'extension et la restriction du sens ainsi que l'emprunt.

Le recours à ces matrices, processus, procédés ou moyens néologiques nous a permis de créer en langue cible $66_{3}$ UT dont les spécificités qui frappent l'œil du bantuiste aussi bien à la lecture qu'à l'analyse sont entre autres : une syntaxe fortement elliptique, des tailles diversement surcomposées et une morphologie composite, où abondent de nombreux morphèmes affixés et des déterminants aux nuances diversifiées.

Ces spécificités propres aux termes dénommant les EMB en ciluba constituent ainsi le deuxième de trois principaux objectifs que nous visions à travers la présente recherche. Soulignons qu'elles sont en parfaite adéquation avec une conviction partagée par beaucoup de lexicologues qui soutiennent que le lexique d'une langue est structuré. C'est ce que nous avons pu comprendre à travers ces propos de Polguère (cité dans L'Homme, 2004 : 83) et par lesquels nous avons introduit notre conclusion générale : Le lexique est un gigantesque réseau où tout se tient. Il suffit de " tirer " sur une lexie de ce réseau pour que vienne avec elle toute une série d'autres lexies auxquelles elle semble attachée par des liens parfois très subtils".

Ce travail aura montré également que la connaissance ainsi que la maîtrise des matrices terminogéniques ou procédés néologiques demeurent sans conteste les deux clés de succès de toute activité néologique dans une entreprise de localisation qui vise l'appropriation des technologies occidentales dans les langues en développement. C'est dans ce même ordre d'idées que doivent êtres compris ces propos de Edema (2008 : 99) lorsqu'il écrit : " La connaissance approfondie de ces procédés est indispensable pour mener à bien toute activité de création de néologie dans le cadre d'un projet d'aménagement linguistique pour instrumentaliser une langue ".

\subsection{Contribution}

Cette recherche avait pour objet l'étude de la néologie terminologique en bantu à travers un exercice de localisation en ciluba des termes français dénommant les EMB. 
Aux fins de bien la conduire à son terme, nous avons eu recours aux théories linguistiques d'importation occidentale, élaborées à l'origine pour le besoin des langues de grandes audiences en usage dans les pays développés et ces théories ont nécessité au plan méthodologique un effort d'adaptation.

C'est dans ce contexte que nous avons été amené à imaginer la démarche de la " cilubalisation ". Celle-ci se doit d'être notée, au plan de la méthode, comme notre remarquable contribution à la connaissance de la néologie en bantu du fait qu'elle nous a permis d'identifier et d'attester de ces procédés néologiques jusque-là méconnus dans notre langue-cible : la mixation, la dérivation parasynthétique, la composition synaptique ainsi que toutes ses particularités. Cette identification en ciluba de nouveaux procédés néologiques ne pouvait se faire sans la remise en question de théories linguistiques existantes. C'est ce que semble recommander Mbuyi (I983 : I82), lorsqu'il déclare que " si l'on se contente de répéter et d'imiter, sans esprit critique, certaines études antérieures, il y a danger que, peu à peu, l'esprit de découverte s'émousse et s'estompe ".

\subsection{Perspectives à venir}

Il faut poursuivre les activités visant l'instrumentalisation des nos langues nationales et véhiculaires par l'élaboration des vocabulaires techniques. Face à cette entreprise, nous sommes de l'avis de ceux qui pensent que seule une langue instrumentalisée est à même de permettre une bonne communication de l'information qui est la condition sine qua non de tout développement et de nos jours, nul ne saurait contester que l'information constitue la base à partir de laquelle des actions du développement doivent être entreprises.

Nous fondant sur leurs aptitudes à appréhender et à exprimer la modernité, nos langues nationales congolaises, peuvent êtres exploitées par nos pouvoirs publics dans le processus de modernisation et d'équipement de tous nos secteurs de la vie socioculturelle. Dans cette perspective ces procédés néologiques dont il venait d'être question à peine : la mixation, la dérivation parasynthétique, la composition synaptique peuvent sans doute servir d'outils dans la fabrication des vocabulaires techniques dénommant les technologies d'importation et ce, aux côtés de ces autres procédés des plus familiers : l'affixation, l'emprunt et la composition.

\section{Références}

Boutin-Quesnel, R. et al. (1990). "Vocabulaire systématique de la terminologie " Disponible en ligne sur : https://www.oqlf.gouv.qc.ca/ressources/bibliotheque/dictionnaires/voc_systematique_terminologie.pdf. Consulté le 2 novembre 20 ı.

Bouveret, M. (1996). "Néologie et terminologie : production de sens du terme " Thèse de doctorat, Montpellier : Université Paul Valery - Montpellier III, $415 \mathrm{p}$.

Bureau Des Traductions (1985). "Fournitures de bureau. Lexique français - anglais - français ", publié par la Direction de la terminologie, Bureau des traductions, Secrétariat d'État, Ottawa (Ontario), 23 pages.

_ (I985). "Office Supplies Glossary/Fournitures de bureau - lexique ", Ottawa : Direction de la terminologie, $23 \mathrm{p}$.

Cabre, M.T. (2000). "Terminologie et linguistique : la théorie des portes", dans Terminologies Nouvelles, no 21, Terminologie et diversité culturelle, p. I0-I5. 
- (2007). "La terminologie, une discipline en évolution : le passé, le présent et quelques éléments prospectifs ", dans Lexicographie et terminologie : compatibilité des modèles et des méthodes, sous la dir. de L'Homme Marie-Claude et Sylvie Vandaele, Ottawa : Les Presses de l'Université d'Ottawa, p. 79IOg.

Cafe, L. (1999). "La terminologie ; théorie, méthode et applications ", traduction du catalan, adaptée et mise à jour par Monique Cormier et John Humbley, Paris Masson et Armand Colin, Ottawa, les Presses de l'Université d'Ottawa, 1998, 322 p. (Regards sur la traduction), dans Langues et Linguistiques, Université Laval, no 25, p. 245-250.

Corbeil, J.-C \& A. Archambault (1992), "Le Visuel : dictionnaire thématique français ", zè édition, Montréal, Editions Québec/Amérique, $896 \mathrm{p}$.

Corbeil, J.-C. S.d. "La terminologie : une discipline au service d'objectifs multiples ". Disponible en ligne sur: https://www.ehu.es/euskalosasuna/baliabideak/liburutegia/orCorbeil.pdf. Consulté le 27 novembre $201 \mathrm{I}$.

Diki-Kidiri, M. (2000). "Terminologie et diversité culturelle ", dans Terminologies Nouvelles, no 21 : Terminologie et diversité culturelle, p. 5-6.

- (2008). "Le vocabulaire scientifique, dans les langues africaines" Pour une approche culturelle de la terminologie. Paris : Karthala, 299 p.

Dubois, J. (1969). "Lexicologie et analyse d'énoncé ". Dans Cahiers de lexicologie 2 : I 15-126.

Dubuc, R. (1978). "Manuel pratique de terminologie " Montréal : Linguatech, 98 p.

- (1980). "Manuel pratique de terminologie " Conseil International de langue française, ro5 p.

Edema, A.B. (2008). "La construction du signifiant ", dans Le vocabulaire scientifique dans les langues africaines. Pour une approche culturelle de la terminologie, sous la dir. de Diki-Kidiri Marcel, Paris : Karthala, p. 71-99.

Ginguay, M. (1990). "Dictionnaire d'informatique, bureautique, têlématique, anglais - français ", Paris Masson, ıò̀ édition, $260 \mathrm{p}$.

Gouadec, D. (1990). "Terminologie. Constitution des données". Coll. "Afnor Gestion ", Paris : Afnor, 2 I9 p.

Guilbert, L. (1975). "La créativité lexicale ", Paris : Larousse, 285 p.

Gulloton, N. \& H. Cajolet-Laganière (1996). "Le français au bureau ", Les Publications du Québec, 400 pages (Guides de l'Office de la langue française).

Hermans, A. \& A. Vansteelandt (1999). "Néologie traductive ", dans Terminologies nouvelles, $n^{\circ}$ 20, p. 3743.

Hermans, A. (1995). "Néologie traductive " Vade-mecum pour traducteurs, Bruxelles : Centre de terminologie de Bruxelles - Institut Libre Marie Haps, 33 p.

Kadima Batumona Adi, G. (2007). "Lexique français - ciluba - français des équipements et matériels de Bureau " , Coll. "Terminologie \& Développement ", Helsinki : CRIC-CIT, 42 p.

Kreckova, V. (1997). "Les tendances de la néologie terminologique en français contemporain ". Disponible en ligne sur : https://www.phil.muni.cz/plonedata/wurj/erb/volumes-2I-30/kreckova97.pdf. Consulté le 2 janvier 2010.

L'Homme, M.-C. \& S. Vandaele (2007). " Lexicographie et terminologie : disciplines sæurs ou pratiques distinctes ?",dans Lexicographie et terminologie : compatibilité des modèles et des méthodes, sous la dir. de L'Homme Marie-Claude et Sylvie Vandaele, Ottawa : Les Presses de l'Université d'Ottawa, p. I-26.

(2004). "La terminologie : principes et techniques", Montréal : Les presses de l'Université de Montréal, coll. "Paramètres ", 282 p.

Le Petit Larousse Illustré (2009). Paris : "Larousse ". ISBN 978-2-03-58-4070-7, 1812 pages.

Lerat, P. (1995). " Les Langues spécialisées ”, Paris : PUF, linguistique nouvelle, 201 p.

Loubier, C. (1994). "L'aménagement linguistique au Québec. Enjeux et devenir ". Coll. "Langues et sociétés ". Québec : Office de la langue française, $142 \mathrm{p}$. 
Mbuyi, K. (1983). "La composition lexicale en ciluba - Kasayi " Thèse de doctorat, Paris : Université de la Sorbonne nouvelle Paris III, $268 \mathrm{p}$.

Rey, A. (1979). "La Terminologie, noms et notions ". Coll. "Que sais-je? ", Paris : Presse universitaire de France, $127 \mathrm{p}$.

Rondeau, G. (1984). "Introduction a la terminologie " 2ème édition. Quebec : Gaëtan Morin (éd.), 238 p. 